\title{
COMPORTAMIENTO AGRONÓMICO Y COMPOSICIÓN QUÍMICA DE TRES VARIEDADES DE Brachiaria EN DIFERENTES EDADES DE COSECHA
}

\author{
Juan Avellaneda Cevallos ${ }^{1,2}$, Fernando Cabezas Guerrero' ${ }^{1}$,Gustavo Quintana Zamora ${ }^{2}$, Ricardo Luna Murillo ${ }^{1}$ \\ Oziel Montañez Valdez ${ }^{3}$, Italo Espinoza Guerra ${ }^{2}$, Samir Zambrano Montes ${ }^{1}$, Diego Romero Garaicoa ${ }^{1}$, \\ Jorge Vanegas Ruiz ${ }^{1}$ y Edgar Pinargote Mendoza ${ }^{4}$.
}

\begin{abstract}
'Área de Pastos-Forrajes y Rumiología, Unidad de Investigación Cientifica y Tecnológica, Universidad Técnica Estatal de Quevedo. Av. Walter Andrade. Km 1 11/2 vía a Santo Domingo, C.P. 73. Quevedo, Los Ríos, Ecuador. juan_avellaneda@yahoo.com.

${ }^{2}$ Facultad de Ciencias Pecuarias, Universidad Técnica Estatal de Quevedo.

${ }^{3}$ División de Bienestar y Desarrollo Regional, Departamento de Desarrollo Degional, CUSUR (Centro Universitario del Sur), Universidad de Guadalajara, México.

${ }^{4}$ Departamento de Extensión y Transferencia de Tecnología, Universidad Técnica Estatal de Quevedo.
\end{abstract}

\section{RESUMEN}

Se evaluó el efecto de la edad (E) y la variedad (V) sobre: altura de planta $(\mathrm{AP})(\mathrm{cm})$, longitud de raíz $(\mathrm{cm})$ (LR), número de tallos (NT) y hojas (NH), biomasa forrajera $(\mathrm{BF})(\mathrm{kg} \mathrm{MS} \mathrm{ha-1})$, relación hoja tallo (en $\mathrm{n}^{\circ}$ y peso), digestibilidad in situ DISMS, y composición química de brachiaria decumbens, brizantha y pasto mulato (ruzizienzis 44-6 x brizantha cv. Marandú), cortados a los 28, 56, 84 y 112 d. Se empleó un diseño de parcelas divididas. La parcela grande fue la variedad de brachiaria, y la parcela pequeña la edad de cosecha. La $\mathrm{AP}$, no presentó diferencias $(\mathrm{p}>0.05)$ entre variedades. La LR presentó diferencia $(\mathrm{p}<0.05)$ entre variedad, la mejor resultó el pasto mulato para la edad de $28,56,84$ y 112 d. El mayor NT a los 56, 84 y 112 d, y NH por planta hasta los $84 \mathrm{~d}$ lo obtuvo mulato. La biomasa (kg MS ha $\left.{ }^{-1}\right)$ no presentó diferencias significativas $(\mathrm{p}>0.05)$. El pasto mulato presentó la mejor relación hoja/tallo, a los 28,56 y $84 \mathrm{~d}$ y el brizantha a los $112 \mathrm{~d}(\mathrm{p}<0.05)$. La digestibilidad in situ disminuyó al aumentar la edad. El mayor porcentaje de MS y fibra cruda, y menor proteína se presentó a los $112 \mathrm{~d}$.

Palabras claves: Brachiaria, digestibilidad, edad, variedad, parcelas divididas.

\section{ABSTRACT}

The effect of the age (A) and the variety (V) on: plant height $(\mathrm{PH})(\mathrm{cm})$, root longitude $(\mathrm{cm})(\mathrm{RL})$, number of stems (NT) and leaves (NL), forage biomass (FB) ( $\mathrm{kg} \mathrm{MS} \mathrm{ha}^{-1}$ ), relationship leaf stem (in number and weigh), in situ digestibility DISMS, and chemical composition of Brachiaria decumbens, brizantha and mulatto grass (ruzizienzis 44-6 x brizantha cv. Marandú), cut at the 28, 56, 84 and $112 \mathrm{~d}$ was evaluated. A split plot design was used. The main plot was the Brachiaria variety, and the subplots the crop age. The PH, didn't present differences $(p>0.05)$ among varieties. The RL presented difference $(\mathrm{p}<0.05)$ among variety, the best was the mulatto grass for the age 28, 56, 84 and 112 d. The biggest NT to the 56,84 and $112 \mathrm{~d}$, and NL for plant until the $84 \mathrm{~d}$ were obtained for mulatto. The biomass (kg MS ha-1) didn't present significant differences $(p>0.05)$. The mulatto grass presented the best relationship leaf/stem, to the 28,56 and $84 \mathrm{~d}$ and the brizantha to the $112 \mathrm{~d}(\mathrm{p}<0.05)$. The digestibility in situ diminished when increasing the age. The major percentage in MS and raw fiber, and smaller protein was presented to the $112 \mathrm{~d}$.

Key words: Brachiaria, Digestibility, age, variety, split plot design.

\section{INTRODUCCIÓN}

La ganadería en el trópico ecuatoriano se basa en explotaciones de doble propósito, y está limitada por la baja productividad de los pastizales. Según INEC (Instituto Nacional de Estadísticas y Censos, 2001) la superficie dedicada a los pastos cultivados y naturales en la provincia de Los Ríos es aproximadamente de 70,077 y 31,638 hectáreas, respectivamente; estando sembrada la mayor parte del terreno de pastos tradicionales, tales como el saboya (Panicun maximun). Sin embargo, también se cuenta con especies tales

Recibido: Noviembre, 2007. Aceptado: Marzo, 2008. Publicado como ARTÍCULO en Ciencia y Tecnología 1: 87-94. 2008. como: $B$. dictyoneura, $B$. decumbens, $B$. brizantha, $B$. humidicola, B. purpurascens, B. ruzizienzis, B. xaraés y el Brachiaria híbrido mulato (ruzizienzis 44-6 $x$ brizantha cv. Marandú). Por ello, es importante conocer a estas especies forrajeras tropicales, con la finalidad de determinar tanto el patrón de crecimiento, composición química y valor nutritivo, toda vez que el conocimiento del crecimiento del pasto es una herramienta útil para planificar su manejo agronómico. Por ello, se planteó la presente investigación con el fin de evaluar el comportamiento agronómico y la calidad nutritiva de algunas especies de Brachiarias en diferentes estados de madurez. 


\section{MATERIALES Y MÉTODOS}

La investigación se llevó a cabo en la finca experimental "La María", de la Universidad Técnica Estatal de Quevedo, ubicada en el km 7 de la Vía Quevedo-El Empalme. En el Cantón Mocache, Provincia de Los Ríos. Se encuentra a una altura de $73 \mathrm{~m}$ sobre el nivel del mar; y duró 4 meses (marzo-junio). La precipitación anual fue de $1690 \mathrm{~mm}$, y el periodo considerado de seca fue de julio a diciembre (6.34\% de la precipitación anual). El suelo del área experimental es clasificado como franco. Un análisis químico del suelo, antes de la implantación de los pastos, mostró los siguientes resultados: 5.5 de $\mathrm{pH}, 36.0 \mathrm{~g} \mathrm{~kg}^{-1}$ de materia orgánica, $10 \mathrm{ppm}$ de $\mathrm{N}, 9 \mathrm{ppm}$ de P, $0.91 \mathrm{meq} \mathrm{dm}^{-3} \mathrm{de} \mathrm{K}$, Ca meq $\mathrm{dm}^{-3}, 1.4 \mathrm{meq} \mathrm{dm}^{-3} \mathrm{de}$ $\mathrm{Mg}, 2.0 \mathrm{ppm}$ de S, $3.9 \mathrm{ppm}$ de $\mathrm{Zn}, 7.0 \mathrm{ppm}$ de $\mathrm{Cu}, 208$ ppm de Fe, 7.5 ppm de Mn, y 0.11 ppm de B.

Se utilizaron quince parcelas experimentales de $3.50 \mathrm{~m}$ x $4.00 \mathrm{~m}$ donde se encontraban 72 bolsas plásticas por parcela que contenían a las variedades a evaluar. Se evaluó el comportamiento de dos factores: El factor (V), fueron las tres variedades de Brachiarias (V1: Brachiaria Brizantha, V2: Brachiaria Decumbens, V3: Brachiaria hibrido (mulato); y el factor (E), las cuatro edades de cosecha (estados de madurez: 28, 56, 84 y $112 \mathrm{~d}$ ).

\section{Experimento 1}

Se empleó un diseño de parcelas divididas, donde la parcela grande fue las variedades de Brachiaria, y la parcela pequeña las edades de cosecha después de la siembra. Se utilizaron cinco repeticiones por tratamiento. Para la comparación entre las medias de los tratamientos se empleó la prueba de Tukey al 5\% de probabilidad. Para el efecto se usó un procedimiento de los modelos lineales generales (PROC GLM) del SAS (SAS, 1999). El modelo matemático fue: $\mathrm{Y}_{\mathrm{ijk}}=\mu+\mathrm{E}_{\mathrm{i}}+\delta_{\mathrm{ij}}+\mathrm{V}_{\mathrm{j}}+\mathrm{EV}_{\mathrm{ij}}$ $+\epsilon_{\mathrm{ijk}}$, donde: $Y_{\mathrm{ijk}}=$ total de una observación, $\mu=$ media general, $\mathrm{E}_{\mathrm{i}}=$ efecto del factor de la parcela principal (variedad), $\delta_{\mathrm{ij}}=$ error experimental asociado a la parcela grande (error $\mathrm{a}$ ), $\mathrm{V}_{\mathrm{j}}=$ efecto del factor de la subparcela (edad), $\mathrm{EV}_{\mathrm{ij}}=$ interacción entre las edad y la variedad, $\mathrm{y}$ $\epsilon_{\mathrm{ij \textrm {j }}}=$ error experimental asociado a las subparcelas (error b).

La unidad experimental estuvo constituida por 6 bolsas de plástico de $30 \mathrm{~cm}$ de diámetro y $45 \mathrm{~cm}$ de largo que contenían las respectivas variedades de Brachiaria, las mismas que representaban a la parcela pequeña, a la cual se le asignó al azar la fecha de la cosecha $(28,56$, 84, y 112 d). La evaluación de las variables agronómicas se realizó mediante el método destructivo de las plantas, siendo las variables experimentales: altura de planta (AP, cm), la que se midió longitudinalmente con una cinta métrica, desde la superficie del suelo contenido en las bolsas; longitud promedio de la raíz $(\mathrm{cm})$, se efectuó la medición de dieciocho estructuras radicales de la planta y se procedió al cálculo de la longitud promedio; número de tallos y hojas por planta $\left(\mathrm{N}^{\circ}\right)$, se contaron todos los tallos y hojas que poseían las plantas de las 6 bolsas de cada unidad experimental, luego se registró el valor promedio; biomasa forrajera $(\mathrm{BF})(\mathrm{kg}$ $\mathrm{MS} \mathrm{ha}^{-1}$ ), para el cálculo de esta variable se consideró el peso seco de hojas y tallos, las hojas y los tallos fueron pesados después de haber salido de la estufa y se tomó en consideración la distancia de siembra $(0,50 \times 0,50$ $\mathrm{m})$; relación hoja tallo ( $\mathrm{N}^{\mathrm{o}}$ y Peso), tanto el peso como el número de hojas fueron divididos para los tallos, y de esa manera se estableció la relación hoja tallo.

\section{Experimento 2}

Se efectuó el análisis de la composición química mediante el análisis proximal propuesto por la A.O.A.C. (1990). Para la digestibilidad in situ se usó bolsas de nylon de 21.5 x $10.5 \mathrm{~cm}$. Se procedió a pesar $5 \mathrm{~g}$ de forraje molido con criba de $2 \mathrm{~mm}$. Para cada muestra de forraje se preparó tres bolsas por edad y variedad para ser incubadas durante $72 \mathrm{~h}$. Finalmente realizado todo este proceso fueron lavadas con agua corriente, y secadas en una estufa a $65{ }^{\circ} \mathrm{C}$ por $48 \mathrm{~h}$. La diferencia entre el peso de la muestra antes de la degradación y después nos indicó la muestra digerida. Se utilizaron tres toretes Brown Swis, fistulados a nivel del rumen, con un peso promedio de $300 \mathrm{~kg}$. Para el estudio de la digestibilidad in situ se empleó un diseño DCA, dónde se empleó la prueba de Tuckey al 5\% de probabilidad. Para el efecto se usó en procedimiento de los modelos lineales generales (PROC GLM) del SAS (SAS, 1999).

\section{RESULTADOS Y DISCUSIÓN}

\section{Experimento 1}

\section{Efecto de la variedad}

La altura de planta $(\mathrm{cm})$ no presentó diferencias (p>0.05) (Cuadro 1) en las variedades de brachiarias estudiadas; sin embargo, la especie brizantha tuvo mayor altura (73.09), al ser comparada con la decumbens y mulato (72.00 y 69.38 , respectivamente), este resultado puede deberse al hábito de crecimiento de las diferentes especies, toda vez que las especies con crecimiento macolloso (brizantha) suelen presentar mayor altura que las de crecimiento decumbentes y estolonífero; estos datos concuerdan con Pérez et al. (1997), en lo referente al comportamiento de las diferentes especies de brachiarias, ya que observaron 
que la $B$. brizantha, presenta mayor altura $(93.70 \mathrm{~cm})$ al compararla con las de $B$. humidicola y dictyoneura, así mismo, Gómez et al. (2000) encontraron que la altura de diferentes accesiones de B. brizantha (106) fue mayor $(\mathrm{p}<0.05)$ que en las accesiones de $B$. decumbens (93), B. ruziziensis (92) B. humidícola (47).

La longitud de raíz $(\mathrm{cm})$ del pasto mulato (26.92) fue mayor $(\mathrm{p}<0.05)$ (Cuadro 1) que la del brizantha (23.62) y decumbens (22.39); al respecto Newman y Delgado (1999) afirman que en el pasto Buffel (Cenhrus ciliaris L.) la longitud de raíz se hace constante a partir de $\operatorname{los} 35 \mathrm{~d}$, presentando un valor aproximado de 44.73 $\mathrm{cm}$, superando éste al observado en la presente investigación.

El mayor $(\mathrm{p}<0.05)$ (Cuaro 1) número de tallos y hojas por planta se observó en el pasto mulato $(7.55$ y 28.70), seguido por el decumbens (6.55 y 26.60) y brizantha (5.50 y 20.50, respectivamente). En cuanto al número de tallos Newman y Delgado (1999) afirman que el pasto Buffel posee incrementos de tallos y hojas de hasta 7,07 y 27,20 respectivamente; se puede apreciar que el número de hojas está siempre por encima del número de tallos, esto permite observar una arquitectura de hojas que apunta a maximizar el uso de la energía solar.

La biomasa forrajera ( $\mathrm{kg} \mathrm{MS} \mathrm{ha} \mathrm{ha}^{-1}$ ) fue mayor $(\mathrm{p}<0.05)$ (Cuadro 1) para el pasto mulato (2001.60) superando al pasto brizantha (1643.45) y decumbens (1154.40), sin embargo, Vergara y Araujo (2006) encontraron valores de producción de materia seca superiores a la presente investigación para la época seca y lluviosa (2216 y 2640) con B. humidícola pudiéndose deber esto al ecotipo o a la calidad de los suelos.

La mejor $(\mathrm{p}>0.05)$ relación hoja/tallo $\left(\mathrm{N}^{\mathrm{o}} / \mathrm{N}^{\circ}\right)$ (Cuadro 1) la obtuvo el pasto mulato (3.85) luego el decumbens (3.83) y brizantha (3.70), no obstante esta relación en peso $(\mathrm{g} / \mathrm{g})$ fue mayor $(\mathrm{p}<0.05)$ para mulato (1.31) seguido de brizantha (1.15) y decumbens
(0.91); por su parte Vallejos et al. (1989) encontraron en promedio, relaciones hoja/tallo en ecotipos de brachiarias de 1.1, observándose los valores más altos en los ecotipos de B. brizantha, sin embargo, Otoya (1986) encontró valores inferiores a los reportados por Vallejos et al. (1989), pero similares a los encontrados en la presente investigación en los que al pasto $B$. decumbens se refiere.

\section{Efecto de la edad}

A los $112 \mathrm{~d}$ se obtuvieron los mayores valores $(\mathrm{p}<0.05)$ (Cuadro 2), en altura de planta $(102.94 \mathrm{~cm})$, longitud de raíz $(29.07 \mathrm{~cm})$, número de tallos y hojas por planta (10.40 y 44.27, respectivamente), relación hoja tallo en número (4.29) y biomasa $(3419.59 \mathrm{~kg}$ MS ha-1), siendo esto confirmado por Costa y Paulino (1999) quienes evaluaron el desempeño agronómico de genotipos de $B$. humidícola y B. brizantha (Costa y Paulino, 1998) en diferentes edades de corte.

\section{Efecto de la interacción variedad por la edad}

La variable altura de planta $(\mathrm{cm})$ no presentó diferencias $(\mathrm{p}>0.05)$ (Figura 1a) entre variedades para las diferentes edades; no obstante, decumbens presentó la mayor altura a los $28 \mathrm{~d}(33.50)$, brizantha a los 56 y 84 d (68.74; 98.69) y el mulato a los 112 d (106.74) lo que puede deducirse que el pasto mulato es de crecimiento lento, pero supera en altura a decumbens y brizantha a $\operatorname{los} 112 \mathrm{~d}$

La longitud de raíz ( $\mathrm{LR}, \mathrm{cm})$, presentó diferencias $(\mathrm{p}<0.05)$ (Figura 1b) entre variedad por edad, como mejor variedad resultó el pasto mulato para las edades $28,56,84$ y 112 d (20.41; 25.76; 27.64 y 33.89), al compararse con el brizantha decumbens. Newman y Delgado (1999) encontraron valores promedios superiores de longitud de raíz (44.73) en pasto Buffel entre las edades de 35 y $49 \mathrm{~d}$.

Cuadro 1. Efecto de las variedades en el comportamiento agronómico de Brachiarias.

\begin{tabular}{lcrrr}
\hline \multirow{2}{*}{ Variables } & \multicolumn{4}{c}{ Efecto de las variedades } \\
\cline { 2 - 5 } & Decumbens & Brizantha & Mulato & EEM \\
\hline Altura $(\mathrm{cm})$ & $72.00 \mathrm{a}$ & $73.09 \mathrm{a}$ & $69.38 \mathrm{a}$ & 3.44 \\
Longitud de Raíz $(\mathrm{cm})$ & $22.39 \mathrm{~b}$ & $23.62 \mathrm{~b}$ & $26.92 \mathrm{a}$ & 0.87 \\
Tallos por planta $\left(\mathrm{N}^{\circ}\right)$ & $6.55 \mathrm{ab}$ & $5.50 \mathrm{~b}$ & $7.55 \mathrm{a}$ & 0.47 \\
Hojas por planta $\left(\mathrm{N}^{\mathrm{o}}\right)$ & $26.60 \mathrm{ab}$ & $20.50 \mathrm{~b}$ & $28.70 \mathrm{a}$ & 1.93 \\
Biomasa $\left(k g\right.$ MS ha ha $^{-1}$ & $1154.40 \mathrm{~b}$ & $1643.35 \mathrm{ab}$ & $2001.60 \mathrm{a}$ & 190.16 \\
Relación hoja tallo $\left(\mathrm{N}^{\circ}\right)$ & $3.83 \mathrm{a}$ & $3.70 \mathrm{a}$ & $3.85 \mathrm{a}$ & 0.09 \\
\hline Relación hoja tallo $(\mathrm{g})$ & $0.91 \mathrm{~b}$ & $1.15 \mathrm{a}$ & $1.31 \mathrm{a}$ & 0.05 \\
\hline * Promedios en cada fila con letras iguales no difieren estadísticamente (Tukey $\mathrm{p} \leq 0.05)$ &
\end{tabular}


El mayor número de tallos y hojas por planta $(\mathrm{p}<0.05)$ (Figura 1c, d) a los $28 \mathrm{~d}$ lo obtuvo el pasto decumbens $(3.40 ; 9,80)$, a los 56 y $84 \mathrm{~d}$ el pasto mulato (7.20; $9.60 ; 27.20 ; 33.80$, respectivamente) y a los 112 d nuevamente el pasto mulato pero con el mejor número de tallos (11.20) solamente, puesto que el mayor número de hojas se le atribuye a decumbens (48.20). Newman y Delgado (1999) afirman que el número de hojas está siempre por encima del número de tallos a partir del día 28 , lo cuál explicaría una calidad nutritiva mayor en los primeros estadíos.

La biomasa no presentó diferencias significativas ( $>0.05$ ) (Figura 1e), sin embargo B. brizantha (kg MS $\mathrm{ha}^{-1}$ ) presentó el mayor valor a los $28 \mathrm{~d}$ (59.50), el pasto mulato a los 56, 84 y 112 d (1172.00; 2929.75; 3850.67, respectivamente) y el menor valor para decumbens a las cuatro edades estudiadas (49.82; 421.50; 1581.18; 2564.98); en pasto estrella (Cynodon nlemfuensis), Fernández et al. (1991) afirman que la producción de materia seca se incrementó con la edad tanto en el periodo de lluvia como en el de sequía, lo cual concuerda con los datos anteriores, así mismo Cuadrado et al. (2004) al estudiar brizantha y decumbens en época lluviosa y seca encontraron valores promedios de materia seca a los 24 días del rebrote de 3316 y $20719 \mathrm{~kg} \mathrm{ha}^{-1}$ valores que superan a los obtenidos en el presente trabajo, debido probablemente a que se efectuó el establecimiento de éstas por medio de semilla y los estudiados por Cuadrado et al. (2004) se refieren a después de un corte de igualación.

En cuanto a la relación hoja/tallo en número por planta $(\mathrm{p}<0.05)$, a los 28,56 y $84 \mathrm{~d}$ el pasto mulato fue el mejor $(\mathrm{p}<0.05)(2.09 ; 1.85 ; 0.77)$ y el brizantha a los $112 \mathrm{~d}(0.80)$, la misma variable con relación al peso $(\mathrm{g})$ presentó valores a los $28 \mathrm{~d}$ para el pasto brizantha (3.72) y el decumbens a los 56, 84 y 112 d (4.23; 3.59; 4.59, respectivamente) (Figura 1f, g), Romero et al. (2003) aseguran haber evaluado el pasto brizantha y decumbens sin fertilización en la cual halló valores significativos $(1.73 ; 1.43)$ a la cuarta semana del rebrote, sin embargo aquellos a los que aplicó fertilización (roca fosfórica) resultaron con valores bajos. Esta situación se explica por que en presencia de fertilizantes se produce una mayor elongación de los tallos y aunque hay seguramente incrementos en la producción foliar, no es menos cierto que la senescencia de hojas es también importante, razón por la cuál prevalece la cantidad de tallos, esto ha sido demostrado por Romero et al. (2003).

\section{Experimento 2}

\section{Efectos de la variedad}

El contenido de materia seca (\%) del heno de pasto brizantha $(90.42)$ fue mayor $(\mathrm{p}>0.05)$ que la de mulato (90.28) y decumbens (90.18). En cuanto a ceniza y proteína bruta fue mayor $(\mathrm{p}>0.05)$ en la variedad decumbens $(10.51 ; 12.99)$ al ser comparado con brizantha $(10.46 ; 11.90)$ y mulato $(11.42 ; 11.53)$ (Cuadro 3). Arias y Hernández (2002) afirman no haber encontrado diferencias significativas $(p>0.05)$ en dos fracciones (hojas y planta integral) de brachiaria humidícola lo cuál concuerdan con los datos hallados en la presente investigación. No obstante los mismos autores atribuyen que la disminución de la proteína esta vinculada con las variaciones climáticas y crecimiento de la planta, y la calidad de la ceniza esta determinada por el consumo permanente de nutrientes por parte de la planta sin ser sustituidos (Cuadro 3).

El extracto etéreo $(\%)$ fue mayor $(\mathrm{p}<0.05)$ para el pasto brizantha (2.29) superando a mulato (2.08) y decumbens (2.06), sin embargo Arias y Hernández (2002) al comparar hojas con muestra integral en $B$. humidicola, encontraron que éstas últimas superan a las hojas en cuanto a extracto etéreo, esto se debe probablemente a la lignificación del tallo presente en la muestra integral. Los pastos evaluados no presentaron diferencias significativas $(\mathrm{p}<0.05)$ con relación a la energía bruta ( $\left.\mathrm{kcal} \mathrm{g}^{-1}\right)$, mulato (3.91), decumbens (3.79) y brizantha (3.77) (Cuadro 3).

Cuadro 2. Efecto de la edad de cosecha en el comportamiento agronómico de Brachiarias.

\begin{tabular}{|c|c|c|c|c|c|}
\hline \multirow{2}{*}{ Variables } & \multicolumn{5}{|c|}{ Efecto de las edades } \\
\hline & 28 & 56 & 84 & 112 & EEM \\
\hline Altura (cm) & $27.50 \mathrm{~d}$ & $64.92 \mathrm{c}$ & $90.61 \mathrm{~b}$ & $102.94 \mathrm{a}$ & 2.92 \\
\hline Longitud de Raíz (cm) & $18.21 \mathrm{c}$ & $25.51 \mathrm{~b}$ & $24.47 \mathrm{~b}$ & $29.07 \mathrm{a}$ & 0.81 \\
\hline Tallos por planta $\left(\mathrm{N}^{\circ}\right)$ & $2.67 \mathrm{~d}$ & $4.87 \mathrm{c}$ & $8.20 \mathrm{~b}$ & $10.40 \mathrm{a}$ & 0.50 \\
\hline Hojas por planta $\left(\mathrm{N}^{\circ}\right)$ & $8.60 \mathrm{~d}$ & $18.67 \mathrm{c}$ & $29.53 b$ & $44.27 \mathrm{a}$ & 2.20 \\
\hline Biomasa (kg MS ha $\left.{ }^{-1}\right)$ & $54.40 \mathrm{c}$ & $707.40 \mathrm{c}$ & $2217.90 \mathrm{~b}$ & $3419.59 \mathrm{a}$ & 185.16 \\
\hline Relación hoja tallo $\left(\mathrm{N}^{\circ}\right)$ & $3.44 \mathrm{~b}$ & $3.90 \mathrm{ab}$ & $3.55 \mathrm{~b}$ & $4.29 \mathrm{a}$ & 0.06 \\
\hline Relación hoja tallo (g) & $1.82 \mathrm{a}$ & $1.34 \mathrm{~b}$ & $0.70 \mathrm{c}$ & $0.64 \mathrm{c}$ & 0.03 \\
\hline
\end{tabular}

* Promedios en cada fila con letras iguales no difieren estadísticamente (Tukey $\mathrm{p} \leq 0.05$ ) 


\section{Efecto de la edad}

El mayor $(\mathrm{p}<0.05)$ porcentaje de proteína se obtuvo a los $28 \mathrm{~d}$, sin embargo disminuyó a medida que la edad aumenta ( $56 \mathrm{~d}, 10.69 ; 84$ d, 8.24 y 112 d, 7.49). Ramírez et al. (2004) asegura que la proteína disminuye a medida que aumentan los días, esta disminución se debe a un descenso de la actividad metabólica de los pastos a medida que avanza la edad del rebrote. Los valores encontrados en cenizas durante las dos primeras edades (28 d, 12.75 y $56 \mathrm{~d}, 11.76)$ fueron altamente significativos $(\mathrm{p}<0.05)$, no obstante estas decrecieron paulatinamente a partir del día $84(9.45)$ y $112(9.22)$ (Cuadro 4).
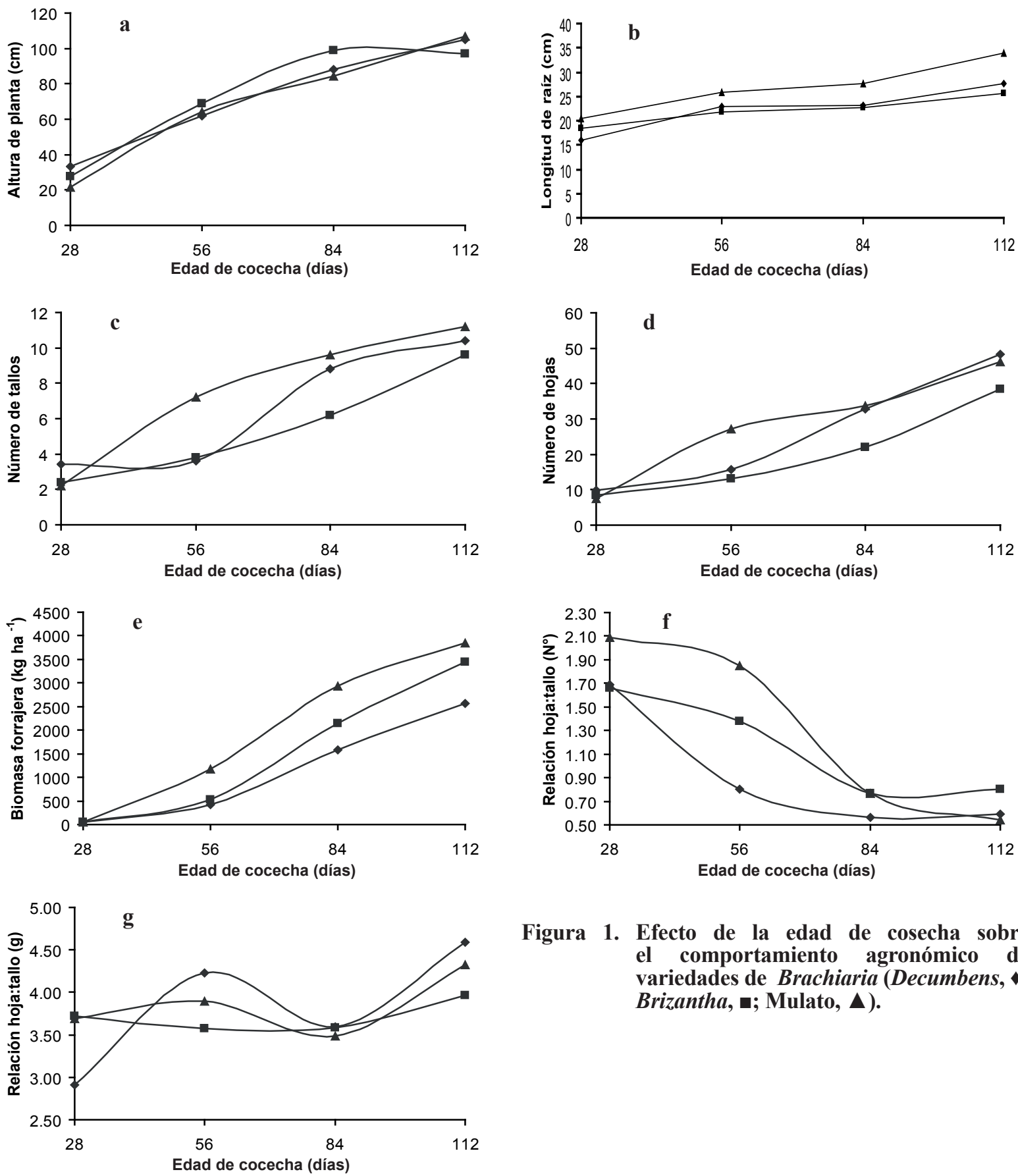

Figura 1. Efecto de la edad de cosecha sobre el comportamiento agronómico de variedades de Brachiaria (Decumbens, ४; Brizantha, $\square$; Mulato, $\Delta$ ). 
Cuadro 3. Efecto de las variedades en la composición química de heno de Brachiarias.

\begin{tabular}{lcccc}
\hline \multirow{2}{*}{\multicolumn{1}{c}{ Variables }} & \multicolumn{4}{c}{ Efecto de las variedades } \\
\cline { 2 - 5 } & Decumbens & Brizantha & Mulato & EEM \\
\hline Humedad total (\%) & $9.78 \mathrm{a}$ & $9.57 \mathrm{a}$ & $9.71 \mathrm{a}$ & 0.09 \\
Materia seca (\%) & $90.18 \mathrm{a}$ & $90.42 \mathrm{a}$ & $90.28 \mathrm{a}$ & 0.08 \\
Ceniza (\%) & $10.51 \mathrm{a}$ & $10.46 \mathrm{a}$ & $11.42 \mathrm{a}$ & 0.26 \\
Proteína bruta (\%) & $12.99 \mathrm{a}$ & $11.90 \mathrm{a}$ & $11.53 \mathrm{a}$ & 0.43 \\
Fibra cruda (\%) & $28.91 \mathrm{a}$ & $28.28 \mathrm{a}$ & $28.56 \mathrm{a}$ & 0.49 \\
Extracto etéreo (\%) & $2.06 \mathrm{~b}$ & $2.29 \mathrm{a}$ & $2.08 \mathrm{~b}$ & 0.04 \\
Energía bruta (kcal g-1) & $3.79 \mathrm{a}$ & $3.77 \mathrm{a}$ & $3.91 \mathrm{a}$ & 0.12 \\
\hline
\end{tabular}

* Promedios en cada fila con letras iguales no difieren estadísticamente (Tukey $\mathrm{p} \leq 0.05$ )

Cuadro 4. Efecto de la edad de cosecha en la composición química de heno de Brachiarias.

\begin{tabular}{lrrrrc}
\hline \multirow{2}{*}{ Variables } & \multicolumn{5}{c}{ Efecto de las edades } \\
\cline { 2 - 6 } & \multicolumn{1}{c}{56} & \multicolumn{1}{c}{84} & 112 & EEM \\
\hline Humedad total (\%) & $10.51 \mathrm{a}$ & $9.87 \mathrm{~b}$ & $8.87 \mathrm{~d}$ & $9.50 \mathrm{c}$ & 0.07 \\
Materia seca (\%) & $89.48 \mathrm{~d}$ & $90.12 \mathrm{c}$ & $91.13 \mathrm{a}$ & $90.45 \mathrm{~b}$ & 0.07 \\
Ceniza (\%) & $12.75 \mathrm{a}$ & $11.76 \mathrm{a}$ & $9.45 \mathrm{~b}$ & $9.22 \mathrm{~b}$ & 0.27 \\
Proté́na bruta (\%) & $12.15 \mathrm{a}$ & $10.69 \mathrm{~b}$ & $8.24 \mathrm{c}$ & $7.49 \mathrm{c}$ & 0.39 \\
Fibra cruda (\%) & $18.99 \mathrm{~b}$ & $31.71 \mathrm{a}$ & $31.20 \mathrm{a}$ & $32.45 \mathrm{a}$ & 0.46 \\
Extracto etéreo (\%) & $3.48 \mathrm{a}$ & $1.87 \mathrm{~b}$ & $1.62 \mathrm{c}$ & $1.61 \mathrm{c}$ & 0.05 \\
Energía bruta (kcal g-1) & $3.98 \mathrm{a}$ & $4.03 \mathrm{a}$ & $3.80 \mathrm{a}$ & $3.79 \mathrm{a}$ & 0.14 \\
\hline
\end{tabular}

* Promedios en cada fila con letras iguales no difieren estadísticamente (Tukey $\mathrm{p} \leq 0.05$ )

La fibra presenta diferencias altamente significativas $(\mathrm{p}<0.05)$ para los días 56 (31.71), 84 (31.20) y 112 (32.45) en comparación con el día 28 (18.99) (Cuadro 4). Ramírez et al. (2004), encontró en pasto decumbens que el contenido de fibra aumenta al avanzar la edad existiendo diferencias significativas entre los $30 \mathrm{~d}$ y otras edades $(45,60,75)$. El mayor contenido de extracto etéreo $(\mathrm{p}<0.05)$ se obtuvo a los $28 \mathrm{~d}$ (3.48) en comparación con las edades 56, 84 y 112 d ( $1.87 ; 1.62$ y 1.61 , respectivamente). La energía bruta no presentó diferencias significativas $(\mathrm{p}>0.05)$, pero el mayor porcentaje de energía se lo obtuvo a los 56 d (4.03), seguido de 28 (3.68), 84 (3.80) 112 d (3.79) (Cuadro 4). Con relación a estas tres últimas variables, autores como Villarreal (1994) coinciden que cuando la edad del pasto se incrementa se produce una disminución progresiva del valor nutritivo.

Efecto de la interacción variedad por edad en la composición química y la digestibilidad in situ

Las variables de la composición química, no presentaron diferencia $(\mathrm{p}>0.05)$ (Figura 2a, b, c, d, e) entre las variedades estudiadas en las diferentes edades evaluadas. Sin embargo, a los $28 \mathrm{~d}$ el mayor porcentaje de digestibilidad in situ de la materia seca la alcanzó brizantha (74.94) ( $>0.05)$ seguido de Mulato $\mathrm{y}$ decumbens ( 74.76 y 73.55 , respectivamente), en cambio a los $56 \mathrm{~d}$ decumbens tuvo un valor de 70.92, mientras que brizantha y mulato se mantuvieron entre un rango de 64.65 a 66.79 (Figura 2f). El día 84 decumbens seguía manteniendo los valores más altos 69.88 mulato 67.24 y brizantha 63.73 y por último a los $112 \mathrm{~d}$ de edad el pasto Brizantha obtuvo los mejores valores (63.20), mulato y decumbens presentaron los menores valores: 60.86 y 58.70 . Fundamentando lo anterior, Vega et al. (2006), manifiestan que la digestibilidad de la materia seca disminuye con el aumento de la edad de la planta. Resultados similares a estos obtuvieron Ramírez et al. (2004), quienes obtuvieron mayor digestibilidad de la materia seca en edades tempranas.

\section{CONCLUSIONES}

Se observó que aunque no en todas las características agronómicas, el pasto mulato (ruzizienzis 44-6x brizantha cv. Marandú) superó a las otras especias de Brachiarias estudiadas. Sin embargo, en la composición química y el valor nutritivo expresado como digestibilidad in situ de la materia seca, no presentó cambios que conduzcan a considerar que esta especie es superior a las otras estudiadas. 

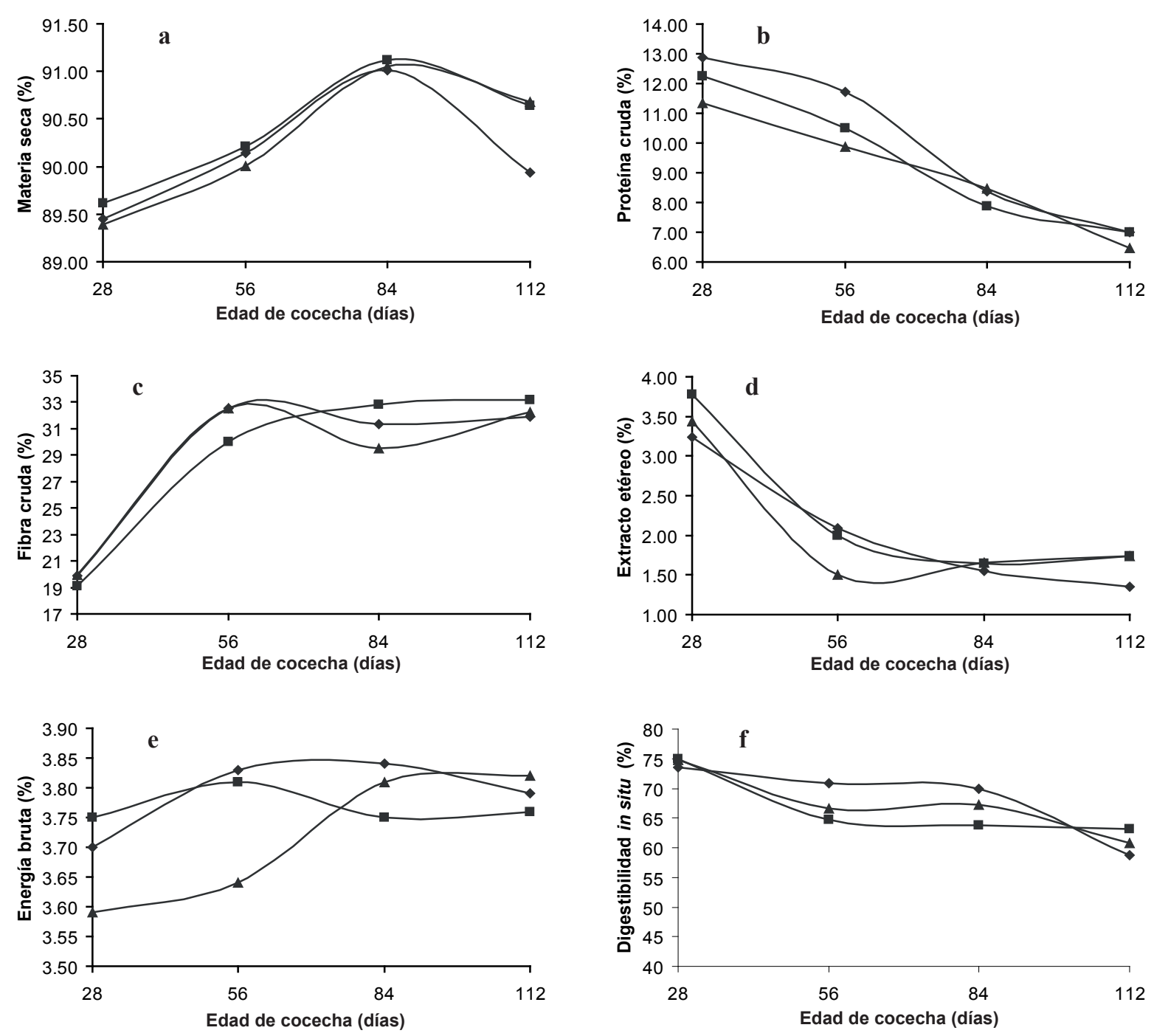

Figura 2. Efecto de la edad de cosecha sobre la composición química y digestibilidad in situ de variedades de Brachiaria (Decumbens, $\diamond$ Brizantha, $\square$; Mulato, $\Delta$ ).

\section{LITERATURA CITADA}

AOAC, 1990. Official Methods of Analysis (15 ${ }^{\text {th }}$ Ed.). Association of Official Analytical Chemists. Arlington, VA, USA. 1990.

Arias, A. y Hernandez, H. 2002. Composición química del pasto aguja (Brachiaria humidicola) sometida a pastoreo en una finca del municipio Guanare estado portuguesa. Rev. Cientif. FCV-LUZ: 12: (Supl. 2) $562-565$.

Costa, N de L., e Paulino, V. 1998. Avaliação agronômica de genótipos de Brachiaria brizantha em diferentes idades de corte. En 35a . Reunido Anual da Sociedade Brasileira de Zootecnia. Botucatu. Anais. Botucatu. Sociedade Brasileira de Zootecnia (SBZ). p: 614616.
Costa, N de L., e Paulino, V. 1999. Desempenho agronômico de genótipos de Brachiaria humidicola en diferentes edades de corte. Pasturas Tropicales. 21:68-71.

Cuadrado, H., Torregraza, L. y Jiménez, N. 2004. Comparación bajo pastoreo con bovinos machos de ceba de cuatro especies de gramíneas del género Brachiaria. MVZ Cordova 9:438-443. (En línea. Consultado el 13 de septiembre de 2007. Disponible en http://apps.unicordoba.edu.co/revistas/revistamvz/ mvz-92/92-4.pdf.

Gómez, A., Rao, M., Beck, F. y Ortíz, M. 2000. Adaptación de una gramínea (C4) y dos leguminosas (C3) forrajeras a un andisol ácido degradado de Colombia. Pasturas Tropicales. 22:19-25. 
Fernández, R., De Chavez, M., Virguez, D. y De Hernandez, M. 1991. Efecto de frecuencia de corte sobre el rendimiento y valor nutritivo del pasto estrella (Cynodon nlemfuensis) en la unidad agroecológica. Zoot. Trop. 9(2): 165-179.

INEC. 2001. Instituto Nacional de Estadísticas y Censos. Pastos Cultivados (en línea) Quevedo EC. Consultado el 04 de octubre 2005. Disponible en: w.w.w.sica.gov.ec/censo/docs/nacionales/tabla29. htm.

Newman, Y. y Delgado, H. 1999. Crecimiento del pasto buffel. 1. Arquitectura de planta. Rev. Fac. Agron. (LUZ) 16:319-326.

Otoya, V. 1986. Efecto de la época del año y días de ocupación en la calidad nutritiva de Brachiaria decumbens. Pasturas Tropicales. 8:2-5.

Pérez, S., Faria, M. y Gonzáles, B. 1997. Evaluación agronómica de gramíneas forrajeras en Carora, estado Lara, Venezuela. Rev. Fac. Agron. (LUZ) 16:621-636.

Ramírez, J., Acosta, I., López, Y., Álvarez, Y. y López, B. 2004. Efecto de la edad de rebrote en el valor nutritivo de dos especies de pastos tropicales (King grass CT 115 y brachiaria decumbens (Consultado el 18 de octubre del 2006) Disponible en http:// www.visionveterinaria.com.

Romero, C., Alfonso, S., Medina, R. y Flores, R. 2003. Evaluación inicial de la fertilización con roca fosfórica entres especies del género brachiaria. Zoot. Trop. 21:183-196.
SAS. 1999. SAS. User's guide: Statistics [CD-ROM Computer file]. Version 8. Statistical Analysis Sistem Institute. Cary, NC.

Vallejos, A., Pizarro, E., Cháves, C., Pezo, D., y Ferreira, P. 1989. Evaluación agronómica de gramíneas en Guapiles, Costa Rica. 1. Ecotipos de Brachiaria. Pasturas Tropicales. 11:2-9.

Vega, E., Ramirez, J., Acosta, L. y Igarza, A. 2006. Rendimiento, caracterización química y digestibilidad del pasto Brachiaria decumbens en las actuales condiciones edafoclimáticas del Valle del Cauto. Rev. Electr. Vet. REDVET. VII (2). (En línea). Consultado el 10 de noviembre 2006 Disponible en http://www.veterinaria.org/revistas/ redvet/n050506.html.

Villareal, M. 1994. Valor nutritivo de gramíneas y leguminosas forrajeras en San Carlos, Costa Rica. Pasturas Tropicales. 16:27-31.

Vergara, J. y Araujo, O. 2006. Producción, composición química y degradabilidad ruminal in situ de brachiaria humidícola (RENDLE) Schweick en el bosque seco tropical. Rev. Cientif. FCV-LUZ. 16: 239-248. 